\title{
A new avenue for treating neuronal diseases: Ceftriaxone, an old antibiotic demonstrating behavioral neuronal effects
}

\author{
Chun-Hwei Tai ${ }^{\mathrm{a}}$, Michele Bellesi ${ }^{\mathrm{b}}$, An-Chih Chen ${ }^{\mathrm{c}}$, Chih-Li Lin ${ }^{\mathrm{d}}$, Hsin-Hua Li ${ }^{\mathrm{d}}$, Pin-Jiun Lin ${ }^{\mathrm{e}}$, \\ Wen-Chieh Liao ${ }^{\mathrm{f}}$, Ching-Sui Hung ${ }^{\mathrm{g}, *}$, Rainer K. Schwarting ${ }^{\mathrm{h}, * *}$, Ying-Jui Ho ${ }^{\mathrm{e}, * * *}$ \\ ${ }^{a}$ Department of Neurology, National Taiwan University Hospital, College of Medicine, National Taiwan University, Taipei, 10002, Taiwan, ROC \\ ${ }^{\mathrm{b}}$ Department of Experimental and Clinical Medicine, Section of Neuroscience and Cell Biology, Universita Politecnica delle Marche, Ancona, Italy \\ ${ }^{c}$ Department of Neurology, Chung Shan Medical University Hospital, Taichung, 402, Taiwan, ROC \\ ${ }^{\mathrm{d}}$ Institute of Medicine, Chung Shan Medical University, Taichung, 402, Taiwan, ROC \\ ${ }^{\mathrm{e}}$ Department of Psychology, Chung Shan Medical University Hospital, Chung Shan Medical University, Taichung, 402, Taiwan, ROC \\ ${ }^{\mathrm{f}}$ Department of Anatomy, Faculty of Medicine, Chung Shan Medical University, Department of Pediatrics, Chung Shan Medical University Hospital, Taichung, 402, Taiwan, \\ $R O C$ \\ ${ }^{\mathrm{g}}$ Occupational Safety and Health Office, Taipei City Hospital, Taipei, 10581, Taiwan, ROC \\ ${ }^{\mathrm{h}}$ Behavioral Neuroscience, Experimental and Biological Psychology, Philipps-Universität Marburg, 35032, Marburg, Germany
}

\section{A R T I C L E I N F O}

\section{Keywords:}

Neurodegenerative disorders

Parkinson's disease dementia

Dementia with Lewy bodies

Alzheimer's disease

Ceftriaxone

\begin{abstract}
A B S T R A C T
Several neurodegenerative disorders, namely Parkinson's disease dementia, dementia with Lewy bodies, and Alzheimer's disease, share common pathophysiological features, such as (1) cognitive deficits, (2) glutamatergic hyperactivity-related excitotoxicity, and (3) deposition of $\alpha$-synuclein ( $\alpha$-syn) and $\beta$-amyloid (A $\beta$ ). Ceftriaxone (CEF) is a well-tested and safe drug that has been used as an antibiotic for several decades. Recent studies have demonstrated the following effects of CEF: (1) increasing glutamate transporter-1 expression and glutamate reuptake and suppressing excitotoxicity, (2) binding well with $\alpha$-syn and inhibition of $\alpha$-syn polymerization, (3) modulating expression of genes related to $A \beta$ metabolism, and (4) enhancing neurogenesis and recovery of neuronal density. In addition, our data revealed that CEF ameliorates seizure and abnormal neuronal firing in the brain. These results suggest the potential of CEF in treating neuronal disorders. This paper addresses the effects and pharmacology of CEF.
\end{abstract}

\section{Introduction to ceftriaxone (CEF)}

CEF is a cephalosporin antibiotic approved for clinical use by the FDA in 1984 as a broad-spectrum antibiotic for infections such as pneumonia [1], bacterial meningitis [2], and gonorrhea [3]. Because CEF has long been used clinically, its safety has been demonstrated $[4,5]$. Recently, neuronal protective effects of CEF were observed in animal models of neurodegenerative disorders, where CEF prevents cognitive and motor deficits; inhibits dopaminergic (DAergic) degeneration in the striatum and substantia nigra pars compacta ( $\mathrm{SNc}$ ); ameliorates cell loss in the hippocampus; restores neuronal density and activity in the striatum, SNc, and hippocampus; and increases neurogenesis in the substantia nigra and hippocampal dentate gyrus (DG).
Increased glutamate reuptake through enhanced glutamate transporter1 (GLT-1) expression, elevation of neurogenesis, and regulation of neuronal electrical activity may underlie the CEF-mediated neuronal and behavioral protections. The results support the use of CEF in patients with neuronal disorders.

Several neurological studies of CEF were inspired by a groundbreaking finding reported by Rothstein et al., who screened antibiotics with the $\beta$-lactam structure from 1040 FDA-approved drugs and observed that CEF is able to promote transcription of the GLT-1 gene and to increase GLT-1 protein levels in astrocytes. CEF enhanced glutamate reuptake, reduced excitotoxicity, exhibited neuronal protection in ischemia, and improves motor function in an animal model of amyotrophic lateral sclerosis (ALS) [6]. Activation of the GLT-1 promotor,

\footnotetext{
* Corresponding author at: Occupational Safety and Health Office, Taipei City Hospital, No.145, Zhengzhou Rd., Datong Dist., Taipei City, 10341, Taiwan, ROC.

** Corresponding author at: Behavioral Neuroscience, Experimental and Biological Psychology, Philipps-Universität Marburg, Gutenbergstr. 18, Center for Mind, Brain, and Behavior (CMBB) 18, 35032, Marburg, Germany.

*** Corresponding author at: Department of Psychology, Chung Shan Medical University Hospital, Chung Shan Medical University, No. 110, Sec. 1, Jianguo N. Rd., Taichung City 402, Taiwan, ROC.

E-mail addresses: bessyhung@gmail.com (C.-S. Hung), schwarti@staff.uni-marburg.de (R.K. Schwarting), yjho@csmu.edu.tw, joshuayjho@gmail.com (Y.-J. Ho).
} 
through the transcriptional nuclear factor kappa B (NFkB) pathway, was observed $48 \mathrm{~h}$ after CEF treatment, which maintained for at least 7 days [6]. After 5 days of CEF (200 mg/kg/day, intraperitoneal injection [IP]) treatment, GLT-1 protein levels increased three-fold compared with controls in the hippocampus and spinal cord. The increased GLT-1 levels are biochemically active because reuptake of $\mathrm{L}-[3 \mathrm{H}]$ glutamate in the cerebral cortex and hippocampus was increased [6]. Further analyses have determined that the fifty percent effective concentration (EC50) of CEF for increasing GLT-1 expression was $3.5 \mu \mathrm{M}$, which is an attainable level $(0.36 \mathrm{mM})$ of CEF in the central nervous system when CEF is used for treating meningitis [7,8]. Rothstein and his colleagues' research is indeed epoch-making. The effects of CEF, promoting GLT-1 expression, increasing glutamate reuptake, and inhibiting neurodegeneration, are quite different from what we known of CEF as an antibiotic. We review the effects of and conclude the mechanism of CEF in the paper.

\section{CEF prevents behavioral and neuronal deficits in the rat model of Parkinson's disease dementia (PDD)}

Several neurological disorders are associated with excessive glutamate levels and deficits of GLT-1 expression [9,10]. Functional interactions between the glutamatergic and dopaminergic (DAergic) systems in the brain regulate motor and cognitive functions. DAergic degeneration causes parkinsonism [11], and atrophy in the hippocampal CA1 area results in anterograde amnesia [12]. Similarly, cell loss in the hippocampal CA1 area has been observed to be accompanied by cognitive deficits in a rat model of PDD [13], because neurogenesis in the hippocampal DG is involved in learning and memory. The hippocampus and the nigrostriatal DAergic system are rich in glutamatergic synapses and are vulnerable to excitotoxic damage; therefore, excessive glutamate release and excitotoxicity in these regions may lead to brain damage and memory and cognition impairments in patients with Parkinson's disease (PD) [14]. Thus, regulation of glutamatergic transmission by enhancing GLT-1 expression could be an ideal pharmacological target to prevent neuronal death and cognitive deficits caused by glutamate excitotoxicity [6,15].

CEF produces a beneficial effect in the PD rat model by regulating glutamatergic activity. In the 1-methyl-4-phenyl-1,2,3,6-tetrahydropyridine (MPTP)-induced PD rat model, decreased GLT-1 expression in astrocytes was observed [13]. CEF treatment (100 or $200 \mathrm{mg} / \mathrm{kg} /$ day, IP) significantly increased GLT-1 expression in astrocyte in the striatum and hippocampus in both sham-operated and MPTP-lesioned rats, irrespective of dose used or whether treatment was started 5 days before or 3 days after the MPTP lesioning [13]. CEF promoted nuclear translocation of p65 and increased the binding of NFKB to the GLT-1 promoter, thus enhancing GLT-1 expression [16]. MPTP lesioning is widely used to induce PD in animal models, because it destroys DAergic neurons in the SNc leading to a loss of DAergic terminals in the striatum. The lesioning also decreases the density of pyramidal neurons in the hippocampal CA1 and DG areas and reduces GLT-1 expression in astrocytes in the striatum and hippocampus $[13,14]$. In addition, MPTP lesioning causes glutamatergic hyperactivity that contributes to excitotoxicity, neurodegeneration, motor function deficits, and impairments in working memory and object recognition, which mimic the pathophysiology of PDD. CEF increased GLT-1 expression, enhanced removal of released glutamate from the synapse, and ameliorated glutamate excitotoxicity [6]. By contrast, intranigral injection of dihydrokainate (DHK), an antagonist of the GLT1 , reversed the protective effects of CEF [17]. Most brain cells express glutamate receptors and are thereby influenced by extracellular glutamate. The brain cells have powerful uptake systems that constantly remove glutamate from the extracellular fluid and thereby limit receptor activation. Although both neurons and astrocytes express glutamate transporters, the GLT-1 in the astrocyte accounts for around $95 \%$ of the total glutamate reuptake activity [18]. Thus, CEF-induced glutamatergic regulation may mainly through the GLT-1 expression in the astrocyte. Furthermore, ischemia increased glutamate release in the cortex and hippocampus, which was suppressed by CEF pretreatment [19].

Immunohistochemical evaluation indicated that CEF treatment significantly inhibited the MPTP-induced decrease of density of DAergic terminals and neurons in the striatum and SNc, respectively. Furthermore, the loss of pyramidal cells in the hippocampal CA1, CA3, and DG areas caused by MPTP was suppressed by CEF treatment $[14,20]$. The number of neural precursor cells and proliferating cells was observed to be reduced in postmortem brains of patients with PD, indicating an impairment of neurogenesis [21]. This was also observed in a PD rat model $[14,22]$. Moreover, we observed that CEF increased the number of newborn cells in the SNr and in the hippocampal DG in the MPTP-induced rat model of PDD [22]. These newborn cells may eventually migrate to the SNc and CA1, respectively, which may explain the recovery of neuronal density in the SNc and hippocampus after CEF treatment. DAergic deficits were considered to be the main pathological cause of PD, which inspired the development of L-dopa and also supported the effects of this drug. However, patients with PD also develop dementia, PDD, and the cognitive deficits are associated with their hippocampal atrophy. L-dopa does not inhibit neurodegeneration in the hippocampus and certainly not block or treat dementia in PD patients. In addition to DAergic degeneration, the hippocampal cell loss mediates PDD. Glutamate-induced excitotoxicity underlie neuronal death in the above two regions. CEF enhances GLT-1 expression, cleans up excess glutamate, prevents excitotoxicity and neurodegeneration, increases new born cells in the brain regions, and improves symptoms of PDD.

CEF affects not only glutamatergic transmission but also the neuronal activity of the brain. The activity of the mitochondrial enzyme, cytochrome oxidase, is used to evaluate metabolic activity of the neuron. MPTP lesioning significantly increased levels of cytochrome oxidase in the subthalamic nucleus (STN). However, this increase was not seen after receiving CEF treatment $[13,14]$. The suppression of STN hyperactivity by CEF was also observed when measuring neuronal activity by using manganese-enhanced magnetic resonance imaging (MEMRI) in vivo [14]. However, MPTP lesioning resulted in decreased neuronal activity in the striatum, cortex, and hippocampus, which was prevented by the treatment with CEF. Furthermore, positive correlations between neuronal activity and density of neurons in the SNc and hippocampus were observed after CEF treatment [14]. Similarly and even more interestingly, we recently determined that CEF treatment suppressed burst discharges in the STN of a PD rat model (Fig. 1). Moreover, Bellesi et al. reported that 8 days of CEF $(200 \mathrm{mg} / \mathrm{kg} /$ day $)$ treatment resulted in a delayed reduction in electroencephalogram (EEG) theta power $(7-9 \mathrm{~Hz})$ in both frontal and parietal areas [23]. Theta oscillations are observed in several areas of the brain, such as the hippocampus and cortical and subcortical structures, which are correlated with long-term potentiation (LTP) [45] and synaptic plasticity [46]. In addition, reduction of theta power reflects lower synchronization of neuronal activity, which may stabilize electrophysiological activity of the brain and may thus be involved in suppression of burst firing and seizures. Changing stimulation frequency from $0.1 \mathrm{~Hz}$ to $1 \mathrm{~Hz}$ for $30 \mathrm{~s}$ caused an increase in field excitatory postsynaptic potentials (fEPSP) amplitude, which was reduced in CEF-treated rats. Furthermore, CEF suppressed LTP and long-term depression (LTD) at synapses of CA3 mossy fibers [24]. Because LTP and LTD are involved in neuronal plasticity, these results suggest a novel mechanism by which CEF regulates neuronal and behavioral functions. It needs further studies to elucidate the mechanism by which CEF affects EEG and LTP/LTD.

\section{CEF may be useful to treat $\alpha$-synuclein ( $\alpha$-syn)- and $\beta$-amyloid} (Aß)-related neuronal disorders

Dementia with Lewy bodies (DLB), PDD, and Alzheimer's disease 
(A) Sham+saline

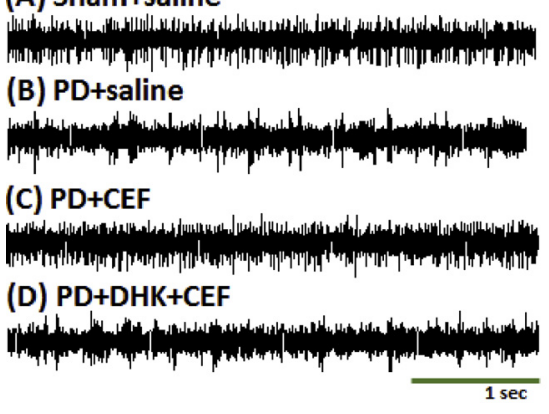

(E)

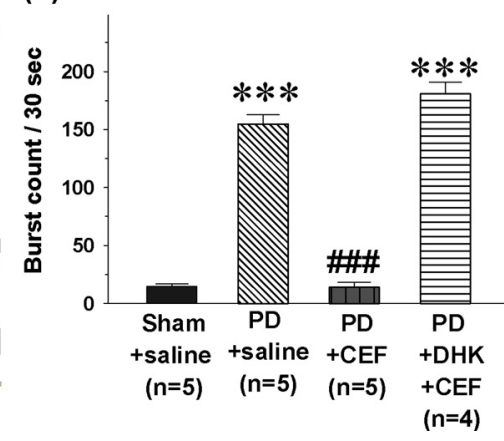

Fig. 1. Effects of ceftriaxone (CEF) on burst discharges in the subthalamic nucleus of a Parkinson's disease (PD) rat model. CEF ( $200 \mathrm{mg} / \mathrm{kg} /$ day, IP) was administered for 2 weeks in the 6-hydroxydopamine-induced PD rat model. (A) The sham + saline group demonstrated a spike firing pattern. (B) Burst discharges were observed in PD rats. (C) Burst discharges were suppressed by CEF treatment. (D) The CEF effect was blocked by dihydrokainate (DHK) $(0.14 \mathrm{mg} / \mathrm{kg} /$ day, IP) treatment. (E) Quantitative results. The data are expressed as the mean \pm SEM. $* * * P<0.001$ compared with the Sham + saline group, \#\#\# $P<0.001$, compared with the $\mathrm{PD}+$ saline group. The experiment and the PD rat model were induced under the approval by the Animal Care Committee of Chung Shan Medical University (IACUC approval No. 1455) and the STN electrical activity was recorded as in our previous reports $[66,67]$.
(AD) are different disorders, but they exhibit common pathophysiological changes, where $\alpha$-synucleinopathies and $A \beta$ cause neuroinflammation and neurodegeneration. Therefore, these changes have been the center of focus in understanding the etiology of these neurodegenerative disorders [25], since elimination of $\alpha$-syn and A $\beta$ may prevent these disorders.

DLB was initially identified as a dementia syndrome with Lewy body pathology. Neurocognitive syndromes occur in patients with DLB when Lewy bodies invade the limbic system and associative neocortices [26]. Cellular pathologies of Lewy bodies, including $\alpha$-syn and A $\beta$ deposition, are hallmarks of DLB [27]. In contrast to DLB, which is characterized by a dominant dementia syndrome followed by parkinsonism, PD is a dominant movement disorder. PD patients also display a dementia syndrome with an initial diagnosis of parkinsonism for more than 1 year, where the cognitive symptoms are thought to occur when Lewy bodies are prevalent in the brain [26]. In addition, recent postmortem studies suggest that as many as one half of AD patients has $\alpha$ syn aggregation in the brain [28,29].

The pathophysiology of DLB is related to the aggregation of Lewy bodies and Lewy neurites that are formed by $\alpha$-syn accumulation [30,31], resulting in neurotoxicity and cell loss in the brain [32-34]. In addition to $\alpha$-syn accumulation, the deposition of extracellular $A \beta$ is also observed in the brain of up to eighty percent of DLB patients [35]. A $\beta$ not only exacerbates neuronal toxicity of $\alpha$-syn but also prolongs the extracellular lifetime of glutamate in the synapse by reducing GLT-1 expression in astrocytes [36]. We reported a method of injection of A $\beta$ and viral vectors with the SNCA gene into the brain to induce the DLB rat model [37]. The DLB rats showed a high level of $\alpha$-syn accumulation in the hippocampal DG area and a lower density of pyramidal neurons in the hippocampal CA1. CEF $(100 \mathrm{mg} / \mathrm{kg} / \mathrm{day}$, IP) treatment corrected the aforementioned neuronal changes and reduced $\alpha$-syn accumulation, indicating that CEF is a potential agent for the treatment of DLB [38]. Neuronal $\alpha$-syn inclusions and pathological $\alpha$-syn transmission play a leading role in the initiation of Parkinson-like neurodegeneration. A recent in vitro study demonstrated that CEF binds to $\alpha$-syn and blocks its polymerization [39]. The binding may underlie the effects of CEF on reducing $\alpha$-syn accumulation and restoring neuronal density and activity in the brain of DLB rats.

Chronic CEF (100 mg/kg/day, IP) administration for 36 days reduced cognitive deficits and ameliorated neurodegenerative changes in the hippocampus in OXYS rats, a model of sporadic AD [40]. The "amyloid cascade hypothesis" proposes that A $\beta$ plays a critical role in the pathogenesis of AD. Modulating the expression of enzymes involved in the metabolism of $A \beta$ has been suggested as an effective strategy for the prevention and therapy of AD. Tikhonova et al. recently determined that CEF $(100 \mathrm{mg} / \mathrm{kg} /$ day, IP) treatment for 36 days affected expression of mRNA, which is involved in $A \beta$ metabolism. CEF diminished the Bace1 mRNA level but augmented Ide, Mme, and Ece1 mRNA levels in the brain of OXYS rats. The Bace1 is involved in the increase of $A \beta$ levels but Ide, Mme, and Ece1 are involved in decrease of A $\mathrm{B}$ levels. Moreover, CEF treatment increased Epo mRNA levels, which are associated with erythropoietin (EPO) expression, neurodevelopment, neurogenesis, and neuroprotection [41]. This is consistent with our previous data illustrating a synergistic effect of combined treatment with CEF and exogenous EPO on neuroprotection as well as cognitive improvements in the MPTP-induced PDD rat model [42]. Accumulation of $\alpha$-syn and $A \beta$ plays an important role in the pathophysiology of DLB, $\mathrm{PDD}$, and $\mathrm{AD}$. A $\beta$ triggers oxidative stress, which stimulates $\alpha$-syn aggregation [43] and deteriorates neurotoxicity [44]. Inhibiting $\alpha$-syn accumulation, enhancing antioxidant activity $[45,46]$, and regulation of $\mathrm{A} \beta$ metabolism, decreasing production and increasing degradation [41], may underlie neuronal and behavioral protections of CEF in the neurological diseases. Further studies are needed to provide support for the $\mathrm{CEF}$ effects on $\mathrm{AD}$ and gene regulations.

\section{Beneficial effects of CEF on movement disorders}

Alexander's disease is a genetic disease characterized by progressive motor deterioration with no cure. A case report indicated that administration of CEF reversed the progression of neurodegeneration in a patient with adult-onset Alexander's disease and substantially improved her quality of life. Before CEF therapy, in a 2-year period, gait ataxia and dysarthria worsened from mild to marked; palatal myoclonus spread from the soft palate to lower facial muscles; and the patient complained of oscillopsia. After 4 years of CEF therapy ( $2 \mathrm{~g} /$ day, IV, for 3 weeks monthly during the initial 4 months, then for 15 days monthly), gait ataxia and dysarthria were improved, from mild to marked at clinical rating scales. The palatal myoclonus was no longer detectable, and the patient did not complain of oscillopsia and reported a progressively better quality of life [47] (Table 1).

A decrease in GLT1 expression has been reported in motor cortex and spinal cord of patients with ALS [48]. CEF ( $200 \mathrm{mg} / \mathrm{kg} /$ day, IP) treatment for 7 days increased muscle grip and reduced the loss of body weight in the animal model of ALS (G93 A SOD1 mice). Two weeks of CEF treatment reduced the death of spinal motor neurons and the gliosis of nerve tissue and improved the survival rate of the ALS animals [6]. Moreover, ALS Functional Rating Scale - Revised (ALSFRS-R) scores declined more slowly in patients with ALS who received CEF treatment ( $4 \mathrm{~g} /$ day, through a central venous catheter for 6 months) than in those on placebo in a large-scale clinical trial involving 514 ALS patients [49]. Notably, restoration of neuronal connections between CNS and PNS may also contribute to CEF effects because increased numbers of corticospinal tract axons and restoration of hind limb motor function were observed after 7 days of CEF $(200 \mathrm{mg} / \mathrm{kg} /$ day, IP) treatment in rats with spinal cord injuries [50].

\section{Beneficial effects of CEF on ischemia, pain, and seizure}

Disruption of homeostasis of glutamatergic neurotransmission, causing excitotoxicity and cell death, plays a pathophysiological role in cerebral ischemia. Middle cerebral artery occlusion increased glutamate release and suppressed astrocytic GLT-1 expression in the frontal cortex 
Table 1

Effects of ceftriaxone on neurological diseases.

\begin{tabular}{|c|c|c|}
\hline Disease & CEF effects & References \\
\hline \multirow[t]{5}{*}{ PD/PDD } & $\begin{array}{l}\text { CEF increased GLT- } 1 \text { expression in astrocyte in the striatum and hippocampus and suppressed hyperactivation } \\
\text { of cytochrome oxidase in the STN in the MPTP-induced PD rat model. }\end{array}$ & [13] Hsu et al., 2015 \\
\hline & CEF inhibited cell loss in the hippocampus and the DAergic system in the MPTP-induced PD rat model. & [14] Weng et al., 2016 \\
\hline & $\begin{array}{l}\text { PD rats showed hyperactivity in the STN but decreased neuronal activity in the striatum, cortex, and } \\
\text { hippocampus, which were ameliorated by CEF treatment, measured by using MEMRI. }\end{array}$ & [20] Ho et al., 2014 \\
\hline & $\begin{array}{l}\text { CEF increased the number of newborn cells in the SNr and in the hippocampal DG in the MPTP-induced rat } \\
\text { model of PDD. }\end{array}$ & [22] Hsieh et al., 2017 \\
\hline & CEF suppressed burst discharges in the STN of PD rat model. & The present paper (Fig. 1) \\
\hline \multirow{4}{*}{$\begin{array}{l}\alpha \text {-Syn and } A \beta \text {-related } \\
\quad \text { disorders }\end{array}$} & CEF corrected neuronal loss and reduced $\alpha$-syn accumulation in the hippocampus in a DLB rat model. & [38] Ho et al., 2018 \\
\hline & CEF binds to $\alpha$-syn and blocks its polymerization. & [39] Ruzza et al., 2014 \\
\hline & $\begin{array}{l}\text { CEF reduced cognitive deficits and ameliorated neurodegenerative changes in the hippocampus in OXYS rats, a } \\
\text { model of sporadic AD. }\end{array}$ & [40] Tikhonova et al., 2017 \\
\hline & In the OXYS AD rat model, $\mathrm{CEF}$ regulated gene expressions that are involved in $\mathrm{A} \beta$ metabolism and EPO levels. & [41] Tikhonova et al. 2018 \\
\hline \multirow[t]{3}{*}{ Movement disorders } & CEF improved gait ataxia, dysarthria, and myoclonus in a patient with Alexander's disease. & [47] Sechi et al., 2013 \\
\hline & $\begin{array}{l}\text { CEF reduced the death of spinal motor neurons and the gliosis of nerve tissue and improved the survival rate in } \\
\text { an ALS animal model. }\end{array}$ & [6] Rothstein et al., 2005 \\
\hline & CEF slowed down the decline of motor function in patients with ALS, in a large-scale clinical trial. & [49] Cudkowicz et al., 2014 \\
\hline Ischemia & $\begin{array}{l}\text { CEF restored changes of glutamate release and GLT- } 1 \text { expression in the frontal cortex and hippocampus and } \\
\text { reduced brain infarct volume in ischemia rat model. }\end{array}$ & $\begin{array}{l}\text { [19] Krzyzanowska et al., } 2017 \\
\text { [51] Chu et al., } 2007\end{array}$ \\
\hline Pain & $\begin{array}{l}\text { CEF alleviated allodynia and hyperalgesia and prevented astrocyte activation in rat models of neuropathic } \\
\text { pain, which is associated with spinal site of action. }\end{array}$ & $\begin{array}{l}\text { [15] Ramos et al., } 2010 \\
\text { [52] Frederiksen et al., } 2017 \\
\text { [53] Lin et al., } 2011\end{array}$ \\
\hline Seizure & $\begin{array}{l}\text { CEF decreased clonic and tonic convulsions, prevented the decline of cognitive function, enhanced oxidative } \\
\text { defense activity, and reduced mortality in the PTZ-induced seizure rats. }\end{array}$ & $\begin{array}{l}\text { [54] Soni et al., } 2015 \\
\text { [55] Jelenkovic et al., } 2008 \\
\text { [56] Hussein et al., } 2016 \\
\text { The present paper (Fig. 2) }\end{array}$ \\
\hline
\end{tabular}

Abbreviations: ceftriaxone (CEF), Parkinson's disease dementia (PDD), glutamate transporter-1 (GLT-1), dopaminergic (DAergic), subthalamic nucleus (STN); manganese-enhanced magnetic resonance imaging (MEMRI); $\alpha$-synuclein ( $\alpha$-syn); $\beta$-amyloid (Aß); Alzheimer's disease (AD), erythropoietin (EPO); amyotrophic lateral sclerosis (ALS); pentylenetetrazole (PTZ).

and hippocampus. CEF $(200 \mathrm{mg} / \mathrm{kg} /$ day, IP) pretreatment suppressed these changes in rats, where enhanced GLT-1 mRNA and protein levels were observed after 3 and 5 days of treatment, respectively [19]. CEFpretreated rats displayed a reduction in brain infarct volume, compared with vehicle-pretreated animals at $24 \mathrm{~h}$ post-ischemia; additionally, the rats showed better functional recovery in a limb placing test at day 1 to week 5 after the ischemia [51]. Lower doses of CEF $(20 \mathrm{mg} / \mathrm{kg} /$ day) reduced infarct volumes to a lesser degree. However, CEF administration at $30 \mathrm{~min}$ after ischemia produced no significant reduction in infarct volume, indicating the vital role of GLT-1 expression. CEF upregulates GLT-1 expression and restores glutamate homeostasis, which may promote brain tolerance to ischemia; therefore, CEF may be a compelling candidate for the development of new therapies to combat brain ischemia.

Hyper-glutamatergic activity is associated with chronic pain. CEF alleviated mechanical allodynia and thermal hyperalgesia in a chronic constriction injury (CCI) rat model of neuropathic pain. Daily dosing of CEF (200, 300, and $400 \mathrm{mg} / \mathrm{kg}$ ) reached the same withdrawal threshold levels as before the CCI surgery, after 18, 12, and 7 days of treatment, respectively. This indicates that the dynamic effect of CEF depends not only on dose, but also on the duration of administration [52]. Thus, it seems that dose exposure above a certain threshold is necessary to produce these effects. Higher doses not only induced larger effects, but also sped up their appearance. Seven days of CEF pretreatment (intrathecal injection) attenuated the development of hyperalgesia and allodynia in response to repeated morphine administration and prevented associated astrocyte activation [15]. One week of administration of CEF was successful to mitigate visceral nociception, which was blocked by intrathecal delivery of selective GLT-1 antagonist DHK, suggesting a spinal site of action [53].

Glutamatergic hyperactivity is involved in abnormal neuronal firing and seizure. Our recent study demonstrated that CEF has protective effects on epilepsy and associated cognitive deficits (Fig. 2). Systemic administration of a sub-convulsive dose of pentylenetetrazole (PTZ) $(30 \mathrm{mg} / \mathrm{kg}$ ) every other day for 27 days (14 injections) increased kindling progressively, led to generalized tonic-clonic seizures, and caused impairments in motor coordination, cognitive function, and oxidative defense in the cortex and subcortical region. CEF (100 and $200 \mathrm{mg} / \mathrm{kg}$ ) treatment significantly decreased the mean kindling score and prevented the decline of cognitive function and oxidative defense activity in the PTZ-induced seizure rats [54]. Similarly, CEF (200 mg/ $\mathrm{kg}$ /day) pretreatment for 6 days provided considerable protective effects against PTZ-evoked generalized clonic and tonic convulsions and convulsion-induced mortality [55]. CEF $(200 \mathrm{mg} / \mathrm{kg} / 12 \mathrm{~h})$ treatment for 3 days, starting from the sixth dose of PTZ, significantly ameliorated PTZ-induced convulsions and restored anti-oxidative activity in rats [56]. Lower percentages of EEG spikes were associated with lowered convulsion in CEF (200 and $400 \mathrm{mg} / \mathrm{kg}$ )-treated rats [57]. Furthermore, a noteworthy case report indicated that cefixime, a cephalosporin antibiotic with similar structure with CEF, also produces an anti-seizure effect. The case report featured a 9-year-old boy suffering from autism and generalized tonic-clonic epilepsy who had taken medications without favorable control of his epilepsy. When the patient took cefixime $200 \mathrm{mg}$ /day to control diarrhea, the seizure episodes were dramatically decreased 3 to 5 days after starting the treatment, though there was no change in his anti-epileptic medication regimen [58].

CEF may treat different symptoms (such as motor dysfunction, cognitive impairment, stroke neuronal death, pain, and seizure) of different diseases through some common mechanisms because these diseases have some common pathophysiological features. CEF may be a non-specific mechanism drug that can simultaneously suppress the common features the neurological diseases, thus treating various symptoms of different diseases and showing multifunctional effects.

\section{Mechanisms of CEF effects on neurological disorders}

Although various neurodegenerative diseases have their main causes, many of the diseases have some common pathophysiological features, such as glutamatergic hyperactivity, excitotoxicity, oxidative stress, neuroinflammation, and accumulation of harmful proteins. CEF 
(A)

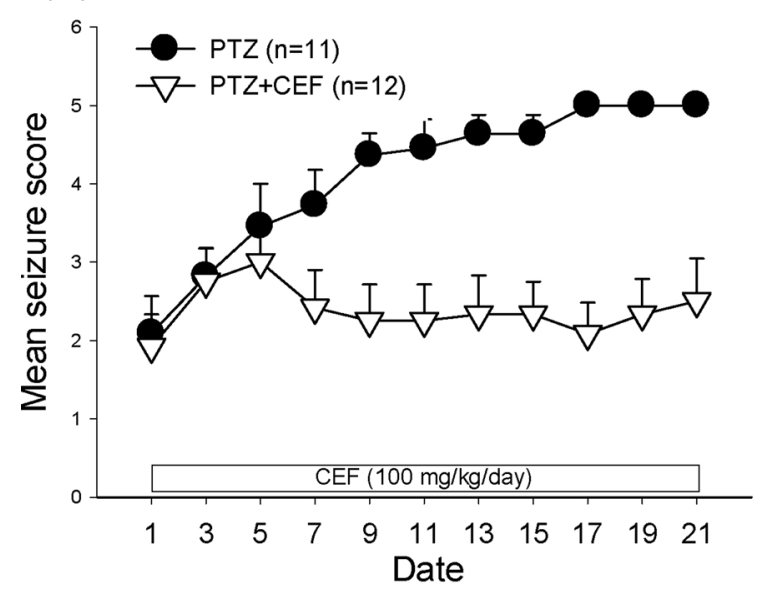

(B)

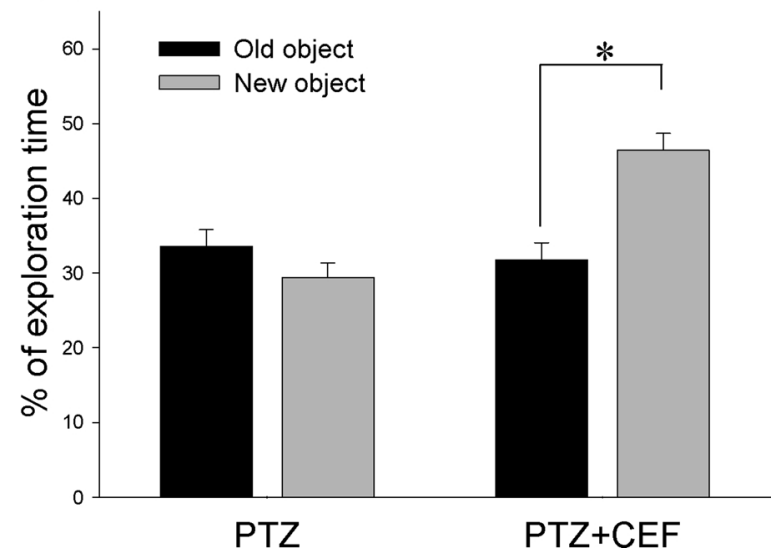

Fig. 2. Effects of ceftriaxone (CEF) on seizures and cognitive function in a pentylenetetrazol (PTZ)-induced seizure rat model. PTZ ( $35 \mathrm{mg} / \mathrm{kg}$, IP) was administered every other day; CEF (100 mg/kg, IP) was administered daily, $6 \mathrm{~h}$ after PTZ injection on the treatment day. Seizure intensity was measured with Racine's convulsion scores [56]. (A) CEF significantly reduced seizure scores starting from day 7. (B) Object recognition was impaired in PTZ-rats, which was prevented in rats receiving CEF treatment, as indicated by a higher percentage of time exploring the new object. The experiment and the seizure rat model were induced under the approval by the Animal Care Committee of Chung Shan Medical University (IACUC approval No. 2083). The object recognition test was performed on days 25 to 27 as in our previous report [22].

exerts several pharmacological effects that inhibits the above pathological features and thus can ameliorate many different neurodegenerative diseases and their symptoms. The above pathophysiological features are involved in neurodegeneration and behavioral dysfunction through downstream biochemical changes. Glutamatergic hyperactivity causes excessive glutamate release; the glutamate activates $N$-methyl-Daspartate (NMDA) receptors and causes $\mathrm{Ca}^{2+}$ overload in the cells, which leads to excitotoxicity and apoptosis. $\mathrm{Ca}^{2+}$ overload can lead to mitochondrial dysfunction, neuronal hyperactivity, seizure, and a consequence of cell death. Cell death induces neuroinflammation, which increases oxidative stress and in turn worsens apoptosis. Aß inhibits GLT-1 expression and suppresses glutamate reuptake, leading to high level of extracellular glutamate. Accumulation of $\alpha$-syn facilitates oxidative stress that gives rise to apoptosis and neuroinflammation. CEF activates NF- $\mathrm{KB}$ and mediates expression of several proteins [59]. CEFinduced increases of GLT-1 expression in the astrocyte enhances glutamate reuptake and lowers extracellular glutamate [6]. CEF regulates expression of genes related to $A ß$ metabolism, reducing production but increasing clearance of Aß [41]. CEF binds directly to the molecular of $\alpha$-syn, inhibiting $\alpha$-syn polymerization and Lewy bodies accumulation [39]. In addition, CEF increases levels of antioxidant enzymes (glutathione and catalase) $[45,46]$ and Bcl2 but decreases caspases 3 and 9 . These effects may mitigate oxidative sttress, apoptosis, and neuroinflammation [46]. Furthermore, CEF promotes levels of brain-derived neurotrophic factor (BDNF) [17] and EPO mRNA [41], which may suppress oxidative stress and be beneficial for neurogenesis as well as neuronal survival. All the aboves are pharmacological effects of CEF, which suppresses neurodegeneration and symptoms of neurological disorders (Fig. 3).

\section{Periodic administration of CEF}

Because CEF has antibacterial activity, using it to treat chronic diseases should not result in drug resistance induced by long-term use. Basic studies have demonstrated that continuous long-term administration of CEF is not necessary because periodic administration is sufficient to produce neuronal and behavioral protections.

Activation of the GLT-1 promotor was observed $48 \mathrm{~h}$ after CEF $(100 \mu \mathrm{M})$ treatment in a cell culture [6]. CEF $(200 \mathrm{mg} / \mathrm{kg} /$ day, IP)-enhanced GLT-1 mRNA and protein expressions were observed after 3 and 5 days of treatment, respectively [51]. Seven days of intrathecal injection with CEF enhanced spinal expression of GLT-1 in naïve rats, which was not observed 7 days after the end of the treatment [15]. Five days of pretreatment with CEF $(200 \mathrm{mg} / \mathrm{kg} /$ day, IP) prevented ischemia-induced behavioral and brain damage [51]. In addition, 7 days of systemic injection of CEF $(200 \mathrm{mg} / \mathrm{kg} /$ day $)$ increased GLT-1 expression in glia, enhanced glutamate reuptake, and displayed neuroprotection in a mouse model of ALS [6]. CEF-induced GLT-1 upregulation persisted for at least 4 days after the end of treatment and returned to baseline 8 days after the end of treatment $[6,60]$.

There is also a delayed emergence of behavioral and electrophysiological (LTD and LTP) changes associated with CEF-induced GLT1 up-regulation, which can be observed after 8 days of CEF treatment. This delay may indicate that synaptic modifications and functional consequences of $\mathrm{CEF}$ require time to effect dynamic changes in neuronal population and lead to behavioral changes [24]. CEF still maintains the same effect after 3 months of continuous treatment. These results indicate that it takes time (e.g., 2-5 days) for CEF to produce its relevant effects, that the effects remain for a period (e.g., 1 week), and that CEF does not quickly develop tolerance [6].

Safety and human dose

CEF is safe and well tolerated. The potential toxicity of CEF $(2 \mathrm{~g} / \mathrm{kg} /$ day) for long-term use has been assessed in SD rats that received CEF administered subcutaneously once daily for 182 consecutive days in order to investigate the delayed onset of any toxicity. No toxicologically meaningful changes were observed. In addition, apart from the decrease in red blood cell count, no CEF-related changes in hematology, coagulation, clinical chemistry, or urine parameters were observed [5].

For conducting clinical trials, data derived from animal models are used to estimate the safe starting dose for human studies. Body surface area (BSA)-based dose calculation is an appropriate method for dose translation across species. For initial clinical trials in healthy adult volunteers, Reagan-Shaw et al. suggested to obtain the human equivalent doses (HED) using the following formula based on BSA normalization of the animal dose [61]: HED $(\mathrm{mg} / \mathrm{kg})=$ rat dose $(\mathrm{mg} /$ $\mathrm{kg}) \times$ (rat $\mathrm{km}$ factor/human $\mathrm{km}$ factor), where the rat $\mathrm{km}$ factor is 6 and the human $\mathrm{km}$ factor is 37 .

The dose of CEF used to treat bacterial infections and meningitis has been reported to be $2 \mathrm{~g}$ /day for 2 months, with no side effects in patients [62]. The recommended CEF routine use for human adults is $1-4 \mathrm{~g} /$ day for seven to 10 days. The effective daily dose of CEF for treating animals in models of neurodegenerative disorder was $200 \mathrm{mg}$ / $\mathrm{kg}$, with no adverse side effects [20,63,64]. Lower doses of CEF (20 and 


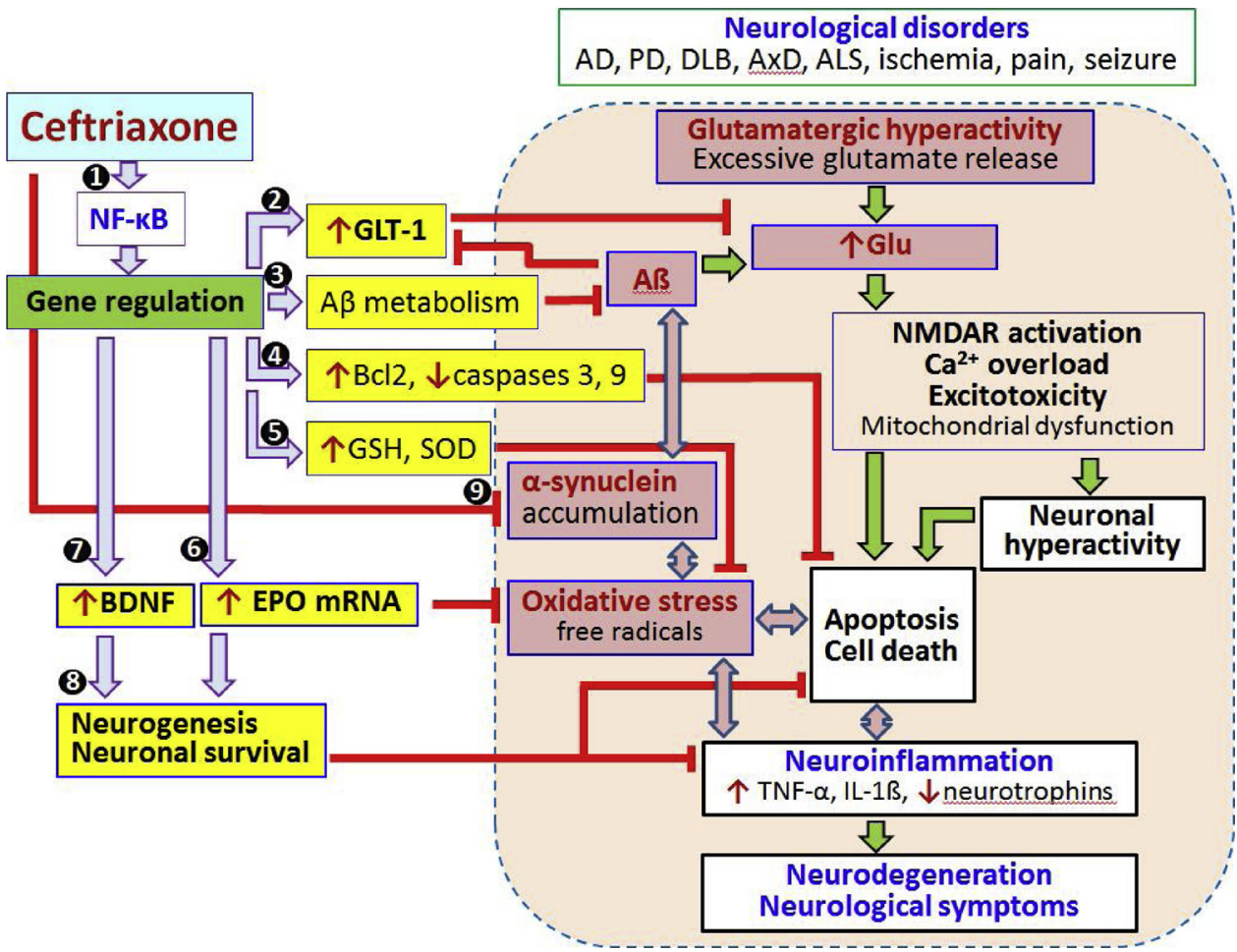

Fig. 3. Mechanisms of neuronal and behavioral protections of ceftriaxone (CEF) in neurological disorders. Some common pathophysiological features are observed in neurological disorders, Alzheimer's disease (AD), Parkinson's disease (PD), dementia with Lewy bodies (DLB), Alexander's disease (AxD), amyotrophic lateral sclerosis (ALS), ischemia, pain, and seizure. These features include glutamatergic hyperactivity, oxidative stress, and accumulation of beta-amyloid protein (Aß) and $\alpha$-synuclein ( $\alpha$-syn), which results in excessive extracellular glutamate (Glu), over activation of N-methyl-D-aspartate receptors (NMDAR), $\mathrm{Ca}^{2+}$ over load, excitotoxicity, mitochondrial dysfunction, neuronal hyperactivity, apoptosis, cell death, neuroinflammation, neurodegeneration, and neurological symptoms. Mechanisms of CEF effects in the disorders include: (1) CEF regulates gene expression and protein levels through activation of NF- $\mathrm{\kappa B}$. (2) $\mathrm{CEF}$ increases expression of glutamate transporter-1 (GLT-1) in the astrocyte, which increases glutamate reuptake and decreases extracellular Glu level. (3) CEF regulates genes related to $A ß$ metabolism, decreasing production of but increasing degradation of $\mathrm{A} \beta .(4,5)$ $\mathrm{CEF}$ increases levels of glutathione (GSH), superoxidase dismutase (SOD), and Bcl2 but decreases caspases 3 and 9, which suppresses



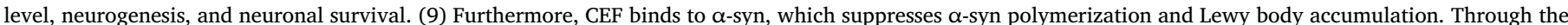

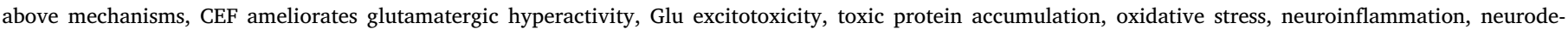
generation, and behavioral symptoms seen in the neurological disorders.

$100 \mathrm{mg} / \mathrm{kg}$ /day) displayed a similar protective effect but to a lesser degree [51]. Notably, CEF at a very low dose of $5 \mathrm{mg} / \mathrm{kg} /$ day also demonstrated neuroprotection and behavioral benefits [42]. Based on the dose translation formula [61], the neuroprotective dose $(200 \mathrm{mg} / \mathrm{kg}$ / day) of CEF in the animal study can be translated to the adult human (body weight $=70 \mathrm{~kg}$ ) equivalent dose as $2.268 \mathrm{~g} /$ day, which is similar to the dosage of CEF when used as an antibiotic (1-4 g/day). Because the up-regulation of GLT-1 expression by CEF is short-lived [6], periodic long-term treatment of CEF will be feasible, in which administration of CEF every other week is suggested. Five days of CEF treatment in the treatment week are necessary to potentiate and prolong its beneficial effects. Using the aforementioned dose (sterilizing dose) and periodic administration (1-week administration and 1-week drug holiday), bacterial resistance can be avoided, although CEF displays antibacterial activity. An international, multi-center phase II clinical trial to assess the efficacy and safety of CEF in patients with mild to moderate PDD, in which CEF is administered periodically and continually, has been approved by the US FDA and is currently being performed in Taiwan [65].

\section{Conclusion}

Several neurodegenerative diseases share common pathophysiological features, such as (1) interaction of $\alpha$-syn and $A \beta$ to form Lewy bodies and (2) glutamate-related neurotoxicity. Recent studies have demonstrated that CEF prevents $\alpha$-syn polymerization and accumulation, inhibits $A \beta$ production, accelerates its elimination, increases GLT1 expression, removes glutamate, reduces excitotoxicity, and enhances neurogenesis. Electrophysiological, neurochemical, and in vivo MEMRI studies suggest that CEF inhibits hyperactivity (e.g., in the STN) but activates neural regions that would otherwise be underactive.

Experiments in animal models of neurodegenerative diseases (e.g., PDD, DLB, AD, ALS, and ischemia) have demonstrated that CEF prevents neurodegeneration, promotes neurogenesis, restores neuronal density, and improves motor and cognitive functions. In addition, CEF demonstrates neurological and behavioral benefits in animal models of epilepsy, pain, and spinal cord injury. CEF slows the progressive deterioration of motor function in patients with ALS and with Alexander's disease and reduces seizure episodes. This evidence suggests that CEF may have potential for the treatment of neurological diseases. Wellcontrolled clinical trials are required to examine the effects in human patients.

Based on the doses used in animal studies, it is estimated that the HED of CEF is $1-2 \mathrm{~g} /$ day for a clinical trial. Because the action of CEF involves the regulation of genes, the activation of promotors, and the production of proteins, it takes several days to produce effects. Once the effects are present, they last for approximately 1 week, so we suggest that CEF should be administered periodically, every other week, and chronically in the clinical trial.

\section{Conflicts of interest}

The authors declare no conflicts of interest for the material in the manuscript.

\section{Acknowledgements}

This work was supported by grants from the Ministry of Science and Technology (MOST 106-2410-H-040-003-MY2; MOST 104-2923-H040-001-MY3; MOST 104-2314-B-040 -007-MY2), Chung Shan Medical University Hospital (CSH-2017-C-007), Taipei City Government (10501-62-046).

\section{References}

[1] S.A. Flanders, V. Dudas, K. Kerr, C.E. McCulloch, R. Gonzales, Effectiveness of ceftriaxone plus doxycycline in the treatment of patients hospitalized with 
community-acquired pneumonia, J. Hosp. Med. 1 (1) (2006) 7-12.

2] A.A. Khrianin, O.V. Reshetnikov, A.D. Anpilogova, [Rocefin (ceftriaxone) in therapy of uncomplicated gonorrhea in males], Antibiotiki i khimioterapiia $=$ Antibiotics and chemoterapy [sic] 51 (8) (2006) 35-37.

[3] E. Molyneux, S.Q. Nizami, S. Saha, K.T. Huu, M. Azam, Z.A. Bhutta, R. Zaki, M.W. Weber, S.A. Qazi, 5 versus 10 days of treatment with ceftriaxone for bacterial meningitis in children: a double-blind randomised equivalence study, Lancet 377 (9780) (2011) 1837-1845.

[4] H. Matsui, M. Komiya, C. Ikeda, A. Tachibana, Comparative pharmacokinetics of YM-13115, ceftriaxone, and ceftazidime in rats, dogs, and rhesus monkeys, Antimicrob. Agents Chemother. 26 (2) (1984) 204-207.

[5] E. Ratti, J.D. Berry, D.J. Greenblatt, L. Loci, A.S. Ellrodt, J.M. Shefner, M.E. Cudkowicz, Preclinical rodent toxicity studies for long term use of ceftriaxone, Toxicol. Rep. 2 (2015) 1396-1403.

[6] J.D. Rothstein, S. Patel, M.R. Regan, C. Haenggeli, Y.H. Huang, D.E. Bergles, L. Jin, M. Dykes Hoberg, S. Vidensky, D.S. Chung, S.V. Toan, L.I. Bruijn, Z.Z. Su, P. Gupta, P.B. Fisher, Beta-lactam antibiotics offer neuroprotection by increasing glutamate transporter expression, Nature 433 (7021) (2005) 73-77.

[7] P.H. Chandrasekar, K.V. Rolston, B.R. Smith, J.L. LeFrock, Diffusion of ceftriaxone into the cerebrospinal fluid of adults, J. Antimicrob. Chemother. 14 (4) (1984) $427-430$.

[8] R. Nau, H.W. Prange, P. Muth, G. Mahr, S. Menck, H. Kolenda, F. Sorgel, Passage of cefotaxime and ceftriaxone into cerebrospinal fluid of patients with uninflamed meninges, Antimicrob. Agents Chemother. 37 (7) (1993) 1518-1524.

[9] P.M. Beart, R.D. O'Shea, Transporters for L-glutamate: an update on their molecular pharmacology and pathological involvement, Br. J. Pharmacol. 150 (1) (2007) 5-17.

[10] A.L. Sheldon, M.B. Robinson, The role of glutamate transporters in neurodegenerative diseases and potential opportunities for intervention, Neurochem. Int. 51 (67) (2007) 333-355.

[11] W. Dauer, S. Przedborski, Parkinson's disease: mechanisms and models, Neuron 39 (6) (2003) 889-909.

[12] S. Zola-Morgan, L.R. Squire, D.G. Amaral, Human amnesia and the medial temporal region: enduring memory impairment following a bilateral lesion limited to field CA1 of the hippocampus, J. Neurosci. 6 (10) (1986) 2950-2967.

[13] C.Y. Hsu, C.S. Hung, H.M. Chang, W.C. Liao, S.C. Ho, Y.J. Ho, Ceftriaxone prevents and reverses behavioral and neuronal deficits in an MPTP-induced animal model of Parkinson's disease dementia, Neuropharmacology 91 (2015) 43-56.

[14] J.C. Weng, M.A. Tikhonova, J.H. Chen, M.S. Shen, W.Y. Meng, Y.T. Chang, K.H. Chen, K.C. Liang, C.S. Hung, T.G. Amstislavskaya, Y.J. Ho, Ceftriaxone prevents the neurodegeneration and decreased neurogenesis seen in a Parkinson's disease rat model: an immunohistochemical and MRI study, Behav. Brain Res. 305 (2016) 126-139.

[15] K.M. Ramos, M.T. Lewis, K.N. Morgan, N.Y. Crysdale, J.L. Kroll, F.R. Taylor, J.A. Harrison, E.M. Sloane, S.F. Maier, L.R. Watkins, Spinal upregulation of glutamate transporter GLT-1 by ceftriaxone: therapeutic efficacy in a range of experimental nervous system disorders, Neuroscience 169 (4) (2010) 1888-1900.

[16] S.G. Lee, Z.Z. Su, L. Emdad, P. Gupta, D. Sarkar, A. Borjabad, D.J. Volsky, P.B. Fisher, Mechanism of ceftriaxone induction of excitatory amino acid transporter-2 expression and glutamate uptake in primary human astrocytes, J. Biol Chem. 283 (19) (2008) 13116-13123.

[17] B. Kaur, A. Prakash, Ceftriaxone attenuates glutamate-mediated neuro-inflammation and restores BDNF in MPTP model of Parkinson's disease in rats, Pathophysiology 24 (2) (2017) 71-79.

[18] N.C. Danbolt, D.N. Furness, Y. Zhou, Neuronal vs glial glutamate uptake: resolving the conundrum, Neurochem. Int. 98 (2016) 29-45.

[19] W. Krzyzanowska, B. Pomierny, B. Bystrowska, L. Pomierny-Chamiolo, M. Filip, B. Budziszewska, J. Pera, Ceftriaxone- and N-acetylcysteine-induced brain tolerance to ischemia: influence on glutamate levels in focal cerebral ischemia, PLoS One 12 (10) (2017) e0186243.

[20] S.C. Ho, C.C. Hsu, C.R. Pawlak, M.A. Tikhonova, T.J. Lai, T.G. Amstislavskaya, Y.J. Ho, Effects of ceftriaxone on the behavioral and neuronal changes in an MPTPinduced Parkinson's disease rat model, Behav. Brain Res. 268 (2014) 177-184.

[21] G.U. Hoglinger, P. Rizk, M.P. Muriel, C. Duyckaerts, W.H. Oertel, I. Caille, E.C. Hirsch, Dopamine depletion impairs precursor cell proliferation in Parkinson disease, Nat. Neurosci. 7 (7) (2004) 726-735.

[22] M.H. Hsieh, W.Y. Meng, W.C. Liao, J.C. Weng, H.H. Li, H.L. Su, C.L. Lin, C.S. Hung, Y.J. Ho, Ceftriaxone reverses deficits of behavior and neurogenesis in an MPTPinduced rat model of Parkinson's disease dementia, Brain Res. Bull. 132 (2017) 129-138.

[23] M. Bellesi, V.V. Vyazovskiy, G. Tononi, C. Cirelli, F. Conti, Reduction of EEG theta power and changes in motor activity in rats treated with ceftriaxone, PLoS One 7 (3) (2012) e34139.

[24] A. Omrani, M. Melone, M. Bellesi, V. Safiulina, T. Aida, K. Tanaka, E. Cherubini, F. Conti, Up-regulation of GLT-1 severely impairs LTD at mossy fibre CA3 synapses, J. Physiol. 587 (Pt. 19) (2009) 4575-4588.

[25] W.S. Kim, K. Kagedal, G.M. Halliday, Alpha-synuclein biology in Lewy body diseases, Alzheimers Res. Ther. 6 (5) (2014) 73.

[26] D.W. Dickson, H. Braak, J.E. Duda, C. Duyckaerts, T. Gasser, G.M. Halliday, J. Hardy, J.B. Leverenz, K. Del Tredici, Z.K. Wszolek, I. Litvan, Neuropathological assessment of Parkinson's disease: refining the diagnostic criteria, Lancet Neurol. 8 (12) (2009) 1150-1157.

[27] G.M. Halliday, J.L. Holton, T. Revesz, D.W. Dickson, Neuropathology underlying clinical variability in patients with synucleinopathies, Acta Neuropathol. 122 (2) (2011) 187-204.

[28] J.B. Toledo, N.J. Cairns, X. Da, K. Chen, D. Carter, A. Fleisher, E. Householder,
N. Ayutyanont, A. Roontiva, R.J. Bauer, P. Eisen, L.M. Shaw, C. Davatzikos, M.W. Weiner, E.M. Reiman, J.C. Morris, J.Q. Trojanowski, Clinical and multimodal biomarker correlates of ADNI neuropathological findings, Acta Neuropathol. Commun. 1 (2013) 65.

[29] I. Mikolaenko, O. Pletnikova, C.H. Kawas, R. O'Brien, S.M. Resnick, B. Crain, J.C. Troncoso, Alpha-synuclein lesions in normal aging, Parkinson disease, and Alzheimer disease: evidence from the Baltimore Longitudinal Study of Aging (BLSA), J. Neuropathol. Exp. Neurol. 64 (2) (2005) 156-162.

[30] B.C. Campbell, Q.-X. Li, J.G. Culvenor, P. Jäkälä, R. Cappai, K. Beyreuther, C.L. Masters, C.A. McLean, Accumulation of insoluble $\alpha$-synuclein in dementia with Lewy bodies, Neurobiol. Dis. 7 (3) (2000) 192-200.

[31] W.J. Schulz-Schaeffer, The synaptic pathology of alpha-synuclein aggregation in dementia with Lewy bodies, Parkinson's disease and Parkinson's disease dementia, Acta Neuropathol. 120 (2) (2010) 131-143.

[32] J. Burre, M. Sharma, T.C. Sudhof, Definition of a molecular pathway mediating alpha-synuclein neurotoxicity, J. Neurosci. 35 (13) (2015) 5221-5232.

[33] M. Ingelsson, Alpha-synuclein oligomers-neurotoxic molecules in parkinson's disease and other Lewy body disorders, Front. Neurosci. 10 (2016) 408.

[34] W.S. Kim, K. Kågedal, G.M. Halliday, Alpha-synuclein biology in Lewy body diseases, Alzheimers Res. Ther. 6 (5) (2014) 73.

[35] S.E. Marsh, M. Blurton-Jones, Examining the mechanisms that link $\beta$-amyloid and a-synuclein pathologies, Alzheimers Res. Ther. 4 (2) (2012) 11.

[36] A. Scimemi, J.S. Meabon, R.L. Woltjer, J.M. Sullivan, J.S. Diamond, D.G. Cook, Amyloid-beta1-42 slows clearance of synaptically released glutamate by mislocalizing astrocytic GLT-1, J. Neurosci. 33 (12) (2013) 5312-5318.

[37] C.L. Lin, Y.S. Cheng, H.H. Li, P.Y. Chiu, Y.T. Chang, Y.J. Ho, T.J. Lai, Amyloid-beta suppresses AMP-activated protein kinase (AMPK) signaling and contributes to alpha-synuclein-induced cytotoxicity, Exp. Neurol. 275P3 (2016) 84-98.

[38] Y.J. Ho, J.C. Weng, C.L. Lin, M.S. Shen, H.H. Li, W.C. Liao, N.M. Tsai, C.S. Hung, T.J. Lai, I.Y. Lee, Ceftriaxone treatment for neuronal deficits: a histological and MEMRI study in a rat model of dementia with Lewy bodies, Behav. Neurol. 2018 (2018) 9. Article ID 4618716

[39] P. Ruzza, G. Siligardi, R. Hussain, A. Marchiani, M. Islami, L. Bubacco, G. Delogu, D. Fabbri, M.A. Dettori, M. Sechi, N. Pala, Y. Spissu, R. Migheli, P.A. Serra, G. Sechi, Ceftriaxone blocks the polymerization of $\alpha$-synuclein and exerts neuroprotective effects in vitro, ACS Chem. Neurosci. 5 (1) (2014) 30-38.

[40] M.A. Tikhonova, S.C. Ho, A.A. Akopyan, N.G. Kolosova, J.C. Weng, W.Y. Meng, C.L. Lin, T.G. Amstislavskaya, Y.J. Ho, Neuroprotective effects of ceftriaxone treatment on cognitive and neuronal deficits in a rat model of accelerated senescence, Behav. Brain Res. 330 (2017) 8-16.

[41] M.A. Tikhonova, T.G. Amstislavskaya, V.M. Belichenko, L.A. Fedoseeva, S.P. Kovalenko, E.E. Pisareva, A.S. Avdeeva, N.G. Kolosova, N.D. Belyaev, L.I. Aftanas, Modulation of the expression of genes related to the system of amyloidbeta metabolism in the brain as a novel mechanism of ceftriaxone neuroprotective properties, BMC Neurosci. 19 (Suppl 1) (2018) 13.

[42] C.K. Huang, Y.T. Chang, T.G. Amstislavskaya, M.A. Tikhonova, C.L. Lin, C.S. Hung, T.J. Lai, Y.J. Ho, Synergistic effects of ceftriaxone and erythropoietin on neuronal and behavioral deficits in an MPTP-induced animal model of Parkinson's disease dementia, Behav. Brain Res. 294 (2015) 198-207.

[43] T. Chan, A.M. Chow, X.R. Cheng, D.W. Tang, I.R. Brown, K. Kerman, Oxidative stress effect of dopamine on alpha-synuclein: electroanalysis of solvent interactions, ACS Chem. Neurosci. 3 (7) (2012) 569-574.

[44] C. Pimentel, L. Batista-Nascimento, C. Rodrigues-Pousada, R.A. Menezes, Oxidative stress in Alzheimer's and Parkinson's diseases: insights from the yeast Saccharomyces cerevisiae, Oxid. Med. Cell. Longev. 2012 (2012) 132146.

[45] R. Bisht, B. Kaur, H. Gupta, A. Prakash, Ceftriaxone mediated rescue of nigral oxidative damage and motor deficits in MPTP model of Parkinson's disease in rats, Neurotoxicology 44 (2014) 71-79.

[46] B. Amin, V. Hajhashemi, K. Abnous, H. Hosseinzadeh, Ceftriaxone, a beta-lactam antibiotic, modulates apoptosis pathways and oxidative stress in a rat model of neuropathic pain, Biomed Res. Int. 2014 (2014) 937568.

[47] G. Sechi, I. Ceccherini, T. Bachetti, G.A. Deiana, E. Sechi, P. Balbi, Ceftriaxone for Alexander's disease: a four-year follow-up, JIMD Rep. 9 (2013) 67-71.

[48] J.D. Rothstein, M. Van Kammen, A.I. Levey, L.J. Martin, R.W. Kuncl, Selective loss of glial glutamate transporter GLT-1 in amyotrophic lateral sclerosis, Ann. Neurol. 38 (1) (1995) 73-84.

[49] M.E. Cudkowicz, S. Titus, M. Kearney, H. Yu, A. Sherman, D. Schoenfeld, D. Hayden, A. Shui, B. Brooks, R. Conwit, D. Felsenstein, D.J. Greenblatt, M. Keroack, J.T. Kissel, R. Miller, J. Rosenfeld, J.D. Rothstein, E. Simpson, N. Tolkoff-Rubin, L. Zinman, J.M. Shefner, Safety and efficacy of ceftriaxone for amyotrophic lateral sclerosis: a multi-stage, randomised, double-blind, placebocontrolled trial, Lancet Neurol. 13 (11) (2014) 1083-1091.

[50] J. Tajkey, A. Biglari, B. Habibi Asl, A. Ramazani, S. Mazloomzadeh, Comparative study on the effects of ceftriaxone and monocytes on recovery after spinal cord injury in rat, Adv. Pharm. Bull. 5 (2) (2015) 189-194.

[51] K. Chu, S.T. Lee, D.I. Sinn, S.Y. Ko, E.H. Kim, J.M. Kim, S.J. Kim, D.K. Park, K.H. Jung, E.C. Song, S.K. Lee, M. Kim, J.K. Roh, Pharmacological induction of ischemic tolerance by glutamate transporter-1 (EAAT2) upregulation, Stroke 38 (1) (2007) 177-182.

[52] K.K. Frederiksen, P. Kristensen, P.H. Honore, G. Gegelashvili, O.J. Bjerrum, Dose and administration-period play a key role in the effect of ceftriaxone on neuropathic pain in CCI-operated rats, Scand. J. Pain 3 (3) (2017) 186.

[53] Y. Lin, K. Roman, K.D. Foust, B.K. Kaspar, M.T. Bailey, R.L. Stephens, Glutamate transporter GLT-1 upregulation attenuates visceral nociception and Hyperalgesia via spinal mechanisms not related to anti-inflammatory or probiotic effects, Pain Res. Treat. 2011 (2011) 507029. 
[54] N. Soni, P. Koushal, B.V. Reddy, R. Deshmukh, P. Kumar, Effect of GLT-1 modulator and P2X7 antagonists alone and in combination in the kindling model of epilepsy in rats, Epilepsy Behav. 48 (2015) 4-14.

[55] A.V. Jelenkovic, M.D. Jovanovic, D.D. Stanimirovic, D.D. Bokonjic, G.G. Ocic, B.S. Boskovic, Beneficial effects of ceftriaxone against pentylenetetrazole-evoked convulsions, Exp. Biol. Med. (Maywood) 233 (11) (2008) 1389-1394.

[56] A.M. Hussein, M. Ghalwash, K. Magdy, O.A. Abulseoud, Beta lactams antibiotic ceftriaxone modulates seizures, oxidative stress and connexin 43 expression in Hippocampus of pentylenetetrazole kindled rats, J. Epilepsy Res. 6 (1) (2016) 8-15.

[57] Y. Uyanikgil, K. Ozkeskek, T. Cavusoglu, V. Solmaz, M.K. Tumer, O. Erbas, Positive effects of ceftriaxone on pentylenetetrazol-induced convulsion model in rats, Int. J. Neurosci. 126 (1) (2016) 70-75.

[58] A. Ghanizadeh, M. Berk, Beta-lactam antibiotics as a possible novel therapy for managing epilepsy and autism, a case report and review of literature, Iran. J. Child Neurol. 9 (1) (2015) 99-102.

[59] K. Kim, S.G. Lee, T.P. Kegelman, Z.Z. Su, S.K. Das, R. Dash, S. Dasgupta, P.M. Barral, M. Hedvat, P. Diaz, J.C. Reed, J.L. Stebbins, M. Pellecchia, D. Sarkar, P.B. Fisher, Role of excitatory amino acid transporter-2 (EAAT2) and glutamate in neurodegeneration: opportunities for developing novel therapeutics, J. Cell. Physiol. 226 (10) (2011) 2484-2493.

[60] M. Bellesi, M. Melone, A. Gubbini, S. Battistacci, F. Conti, GLT-1 upregulation impairs prepulse inhibition of the startle reflex in adult rats, Glia 57 (7) (2009)
$703-713$

[61] S. Reagan-Shaw, M. Nihal, N. Ahmad, Dose translation from animal to human studies revisited, FASEB J. 22 (3) (2007) 659-661.

[62] U. Roelcke, W. Barnett, E. Wilder-Smith, D. Sigmund, W. Hacke, Untreated neuroborreliosis: bannwarth's syndrome evolving into acute schizophrenia-like psychosis. A case report, J. Neurol. 239 (3) (1992) 129-131.

[63] B.R. Miller, J.L. Dorner, M. Shou, Y. Sari, S.J. Barton, D.R. Sengelaub, R.T. Kennedy, G.V. Rebec, Up-regulation of GLT1 expression increases glutamate uptake and attenuates the Huntington's disease phenotype in the R6/2 mouse, Neuroscience 153 (1) (2008) 329-337.

[64] W. Krzyzanowska, B. Pomierny, B. Budziszewska, M. Filip, J. Pera, N-acetylcysteine and ceftriaxone as preconditioning strategies in focal brain ischemia: influence on glutamate transporters expression, Neurotox. Res. 29 (4) (2016) 539-550.

[65] Y.J. Ho, To Assess the Efficacy and Safety of Ceftriaxone in Patients With Mild to Moderate Parkinson's Disease Dementia, BRICEFA20170414 US FDA Approval, (2017) https://clinicaltrials.gov/ct2/results? cond = \&term = BRICEFA20170414\& cntry $=\&$ state $=\&$ city $=\&$ dist $=\&$ Search $=$ Search $)$.

[66] Y.C. Yang, C.H. Tai, M.K. Pan, C.C. Kuo, The T-type calcium channel as a new therapeutic target for Parkinson's disease, Pflugers Arch. 466 (4) (2014) 747-755.

[67] C.H. Tai, Y.C. Yang, M.K. Pan, C.S. Huang, C.C. Kuo, Modulation of subthalamic Ttype $\mathrm{Ca}(2+)$ channels remedies locomotor deficits in a rat model of Parkinson disease, J. Clin. Invest. 121 (8) (2011) 3289-3305. 